\title{
Hodges Conjecture Clay Institute Millennium Problem Solution
}

\author{
Paul T E Cusack, BScE, DULE
}

23 Park Ave., Saint John, NB, Canada E2J 1R2

St-mcihael@hotmail.com

Abstract: Here we consider the Hodge's Conjecture that expresses when a projective manifold coincides with a sum of algebraic cycles. More generally, this entails the convergence of geometry and calculus which has been discussed in this author's previous paper.

Keywords: Hodge’s Conjecture; Polynomials; Analytic sums; Geometry; Calculus.

\section{INTRODUCTION}

In his paper, we consider the Hodge's Conject Clay Institute Millennium Problem solution.That problem is described as follows:

\section{Hodge Conjecture}

Let $\mathrm{X}$ be a non-singular complex projective manifold. Then every Hodge class on $\mathrm{X}$ is a linear combination with rational coefficients of the cosmology classes of complex sub varieties of $\mathrm{X}$.

\section{The Problem}

The Hodge Conjecture addresses the following natural question:

Let $X$ be a projective manifold. Suppose $C$ is a

topological cycle on $X$. When is $C$ homologous to a formal sum of algebraic cycles?

One obvious condition: Since an algebraic cycle is complex, it has even (real) dimension.

A typical issue in geometry: Find a geometric representative of a topological equivalence class of "cycles"

Hodge's proposed characterization of sums of algebraic cycles brings in our final theme:

- Interplay between geometry and calculus

https://www.google.ca/imgres?imgurl=https://www.ma.utexas.edu/users/dafr/HodgeConjecture/jpg/

Slide21.jpg\&imgrefurl=https://www.ma.utexas.edu/users/dafr/HodgeConjecture/netscape_noframes.

html\&h=540\&w=720\&tbnid=2pnWLyrDdf1SiM:\&tbnh=158\&tbnw=211\&usg=_i1tKth78PLTH6hzf2WHWlw0qH0=\&vet=10ahUKEwiwn6iKjIXXhWF64MKHQ-BAaYQ9QEILDAA..i\&docid=gVJyErfNOdd8WM\&sa =X\&ved=0ahUKEwiwn6iKjI_XAhWF64MKHQ-BAaYQ9QEILDAA\#h=540\&imgdii=2pnWLyrDdf1SiM:\&tbnh=15 8\&tbnw=211\&vet=10ahUKEwiwn6iKjI_XAhWF64MKHQ-BAaYQ9QEILDAA..i\&w=720 


\section{Arena for the Hodge Conjecture}

A final generalization in our point of view:

$$
C P^{n} \longrightarrow \text { projective manifold } X
$$

A projective manifold $X$ is defined by (smooth) polynomial equations:

$$
X: P_{1}\left(x^{1}, x^{2}, \ldots, x^{\mathrm{n}}\right)=P_{2}\left(x^{1}, x^{2}, \ldots, x^{\mathrm{n}}\right)=\ldots=P_{\mathrm{s}}\left(x^{1}, x^{2}, \ldots, x^{\mathrm{n}}\right)=0
$$

An algebraic cycle $C$ in $X$ is given by additional polynomial equations:

$$
C: Q_{1}\left(x^{1}, x^{2}, \ldots, x^{\mathrm{n}}\right)=Q_{2}\left(x^{1}, x^{2}, \ldots, x^{\mathrm{n}}\right)=\ldots=Q_{t}\left(x^{1}, x^{2}, \ldots, x^{\mathrm{n}}\right)=0
$$

The Hodge Conjecture concerns algebraic cycles in projective manifolds.

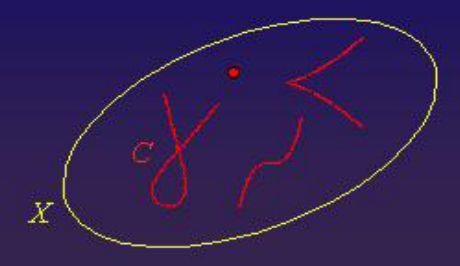

Ibid.

Consider:

CPn $\longrightarrow \gg$ Projected Manifold X

$\mathrm{X}=$ Polynomial $\mathbb{P}^{\mathrm{n}}$

$\mathrm{C}=$ Analytic function $=$ transcendental $(\sin , \mathrm{Ln}, \mathrm{e})$

Let $\mathrm{X}$ be the variable in the Golden Mean Polynomial

$\mathrm{x}^{2}-\mathrm{x}=0$

$x(x-1)=0$

$\mathrm{x}=0$ Trivial

$\mathrm{x}=1$ singular

$\mathrm{X}=2 \quad \mathrm{y}=4$

$\mathrm{X}=3 \mathrm{y}=6$

$\mathrm{X}=4 \quad \mathrm{y}=12$

$\mathrm{X}=5 \mathrm{y}=20$

$\mathrm{X}=6 \mathrm{y}=30$

$\mathrm{X}=-1 \quad \mathrm{y}=2$

$X=-2 \quad y=6$

Etc.

Therefore, roots are always even. 
Hodges Conjecture Clay Institute Millennium Problem Solution

Let $\sum \mathrm{C}=\mathrm{C}$

Eq.(7)

Therefore, the function equals the derivative.

$\int \mathrm{C}=\mathrm{C}^{2} / 2=\mathrm{C}$

Eq (8)

$\mathrm{C}^{2}=2 \mathrm{C}$

$\mathrm{C}=2$

$\mathrm{C} \mathbb{P}^{\mathrm{n}}=2 \mathbb{P}^{\mathrm{n}}=\mathrm{X}$

$2 \mathbb{P}^{\mathrm{n}}=\mathrm{x}^{2}-\mathrm{x}-0$

Eq.(10)

$2 \mathbb{P}^{\mathrm{n}}=1$

$\mathbb{P}^{\mathrm{n}}=1 / 2$

$\mathbb{P}=\sqrt[n]{(1 / 2)}=1 /{ }^{\mathrm{n}} \sqrt{2}$

Let $C^{\prime}=2 / 3 * 2^{3 / 2}=0.4242 \sim \pi-e$

$C=\int\left(\pi-e^{x}\right) d x=x-e^{x}$

$\mathrm{CP}^{\mathrm{n}}=\left(\mathrm{x}-\mathrm{e}^{\mathrm{x}}\right)\left(1 /{ }^{\mathrm{n}} \sqrt{2}\right)=\mathrm{x}^{2}-\mathrm{x}$

Eq.(14)

So, the solution is:

$\mathrm{x}^{2}-\mathrm{x}-\left(\mathrm{x}-\mathrm{e}^{\mathrm{x}}\right)(1 / \sqrt[\mathrm{n}]{\mathbf{2}})=0$

When $n=1$, the last term becomes $\sin 45^{\circ}=\cos 45^{\circ}$

And the natural logarithm function:

when $\mathrm{x}=1, \mathrm{y}=0$

$\&$

$\mathrm{y}^{\prime}=\mathrm{y}=1$

This is the $\ln$ function.

$\sin \mathrm{u}+\mathrm{v}+\mathrm{x}^{2}+\mathrm{y}^{2}=\mathrm{z}^{2}$

For $\mathrm{x}^{2}+\mathrm{y}^{2}=\mathrm{z}^{2}=$ Radius $=1$

When $x=1, y=0$

$\sin \mathrm{u}+\mathrm{v}+\sin \mathrm{x}^{2}+\sin \mathrm{Y}^{2}=\sin (1)$

$0.8415+(-0.8415)+\left(\sin ^{2} 1\right)+\left(\sin ^{2} 0\right)=0.8415$

Eq.(20)

$\sin ^{2} 0=0$

$\sin ^{2} 1=0.8415$

$0.8415-0.8415+0.0+0.8415=0.8415$

$0+0.8415=0.8415$

Eq.(21)

True!

\section{CONCLUSION}

We have worked out a possible set of solutions to the Hodges Conjecture. We have considered Analytic functions, C, summed into the general polynomial, P. This allows us to mold geometry and calculus together. 


\section{REFERENCES}

1. Cusack, P., Astro-Theology, Cusack Universe. J. of Phys. Math. Jan 2016. OMI.

2. Chemistry for Astro-Theology, Fluid Mechanics, Open Access, 4:1.2017.

3. Convergence of Physical Mathematics, Fluid mechanics, Open Access, 4:163. 2017.

4. Convergence and the Grand Unified Theory, J. of Phys. Math. 8:211, 2017.

5. Gravity and Electromagnetism. Fluid mechanics,. Open Access. 4:155. 2017

6. Gravity, Time, Mass and the Superforce. J. of Physical Mathematics. Jan (2018)

7. Naiver-Stokes Clay Institute Millennium Problem Solution. J. of Physical math. 7: 176. 2016.

8. Physics for Astro-theology. Fluid mechanics Open Access. 4: 146. 2016.

9. Riemann Hypothesis Clay Institute Millennium Problem Solution. J ApplComputat. Math 5:317

10. Ross 128 Radio Frequency Detection: An Astro-theology Explanation, (submitted)

11. The Ether, The Universal Material. Fluid Mech Open Acc 3: 1322016.

12. The Fundamental Solution to Economics. Research and Reviews. J. of statistics and Math. Sciences. 2016.

13. The Speed of light and Astro-theology. Fluid Mech Open Acc 4: 148.

14. The Termination of Physical Constants in Proton Mass. Fluid Mech Open Acc 4:150

15. Time Travel for Nostradamus. Research and Reviews. J. of Statistics and math. Sciences. 2016

16. The Western Pacific Biotwang: experiment al Evidence for Astrotheology Cusack's Universe.

17. Uniting Cosmology with Quantum Mechanics. Asian journal of math. Sciences. 1:03, 2017.

18. Universal ODE's and Their Solution. J Phys Math 7: 191. 2016.

19. Universal Soil Mechanics. Asia J. of math. Sciences. 1:03 2017.

20. Universal Structural Mechanics (submitted).

21. Universal Simple Harmonic Impetus. (submitted).

22. What is the Value of the Sqrt (-1)? Research and Reviews. J. of Statisticsand Math. Sciences. 2016.

23. Yang-mills Mass Gap Clay Institute Millennium Problem Solution. J Phys Math 7:171. 2016.

Citation: Paul T E Cusack. "Hodges Conjecture Clay Institute Millennium Problem Solution”. American Research Journal of Mathematics. vol 3, no. 1, 2017, pp. 1-4.

Copyright (C) 2017 Paul T E Cusack, This is an open access article distributed under the Creative Commons Attribution License, which permits unrestricted use, distribution, and reproduction in any medium, provided the original work is properly cited. 